\title{
Chemoradiation with capecitabine and mitomycin-C for stage I-III anal squamous cell carcinoma
}

\author{
Govin Thind', Bal Johal ${ }^{2}$, Matthew Follwell ${ }^{3}$ and Hagen Fritz Kennecke ${ }^{4^{*}}$
}

\begin{abstract}
Background: Standard therapy for patients with stage I-III squamous cell carcinoma (SCC) of the anal canal is chemo-radiotherapy with 5-fluorouracil (5-FU) and mitomycin C (MMC). While there is limited published evidence to substitute capecitabine (CAP) for 5-FU, the objectives of the study were to describe the toxicity, dose intensity and outcomes of a sequential cohort of patients treated with chemo-radiotherapy with CAP and MCC in a population-based setting.

Methods: Patients with stage I-III malignancies of the anal canal referred between February 2010 and March 2012 were included. Dose intensity was calculated by comparing delivered versus planned radiation and chemotherapy treatments and toxicity was retrospectively graded according to standard protocol-specified criteria.

Results: Among 66 eligible patients, median planned dose of radiation was 51.9 Gy over 5.5 weeks, range 25.0 to $63 \mathrm{~Gy}$, and dose intensity was $98 \%$. Median delivered dose of MCC delivered was $12 \mathrm{mg} / \mathrm{m}^{2}$ on day one, week one while median CAP dose was $825 \mathrm{mg} / \mathrm{m}^{2}$ twice daily on radiation days. CAP dose reductions due to toxicity were recorded for 13 patients (20\%). Median follow-up was 20 months and 94\% of patients with squamous cell histology had no evidence of relapse.

Conclusions: Chemo-radiation with CAP plus MMC is well tolerated and may be a reasonable consideration for patients with stage I-III SCC of the anal canal. A range of planned radiation dose was observed and longer follow-up is necessary to ensure that patients who received lower doses of radiation have similar outcomes to those who received larger doses.
\end{abstract}

Keywords: Overall survival, Toxicity, Squamous cell carcinoma, Patient outcomes, Chemoradiation

\section{Background}

Carcinoma of the anal canal is a relatively uncommon malignancy, accounting for approximately 2.5 percent of all gastrointestinal cancers [1]. The incidence of this disease has been on the rise for the past few decades [2], which is thought to be due, in part, to the increased sexual transmission of human papilloma virus (HPV) $[3,4]$.

Squamous cell carcinoma (SCC) of the anal canal remains the only carcinoma of the gastrointestinal tract that is curable without the need for definitive surgery, with 5 year survival rates nearing $90 \%$ for early stage disease

\footnotetext{
* Correspondence: hkennecke@bccancer.bc.ca

${ }^{4}$ Division of Medical Oncology, British Columbia Cancer Agency - Vancouver Center, Vancouver, BC V5Z 4E6, Canada

Full list of author information is available at the end of the article
}

[2,3,5,6]. Treatment regimens for SCC of the anal canal have evolved over the past decades, and studies have included comparisons of radiotherapy alone versus chemoradiation [7-9]; determining treatment benefits of mitomycin C (MMC) [10,11]; and comparisons of MMC with cisplatin [6,12-15]. The accepted current standard regimen for patients with stage I-III SCC of the anal canal is radiotherapy (50.4 Gy) with concurrent infusional 5-fluorouracil (5-FU) administered days one through four during weeks one and five and MMC administered on day one of week one. Substitution of MMC for cisplatin on days one and 29 of radiation has been shown equally efficacious and associated with significantly less hematologic toxicity [6].

Capecitabine (Cap) is an oral fluoropyrimidine shown to be equivalent to infusional 5-FU when given concurrently 
with pelvic irradiation in the neo-adjuvant treatment of rectal adenocarcinoma [16-18]. Unlike 5-FU, which is intravenously infused, Cap is orally administered which provides resource benefits as it is more convenient for patients and staff and does not require the use of a central venous infusional device. There is only limited evidence to support the substitution of infusional 5-FU for capecitabine in the treatment of SCC of the anal canal. In a previously published phase II study, thirty-one patients with stage I-III SCC of the anal canal were treated with continuous radiation, Cap on radiation days and MMC on day 1 [19]. A total of 24 (77\%) of patients had a complete clinical response after four weeks, while 3 (16\%) had a partial response. Three locoregional relapses occurred during the follow up period (median of 14 months) [19].

In this study, a retrospective chart review was conducted of a sequential cohort of patients with stage I-III SCC of the anal canal treated with standard radiation and concurrent Cap and MMC according to a standard province-wide protocol [20]. The objectives of the study were to: (1) describe the dose intensity of radiation, Cap and $\mathrm{MMC}$ by comparing the planned versus the delivered dose of radiation, Cap and MMC; (2) describe treatmentrelated patient toxicities and (3) describe the early outcomes of therapy.

\section{Methods}

\section{Treatment of SCC of the anal canal}

Patients were treated at one of five cancer treatment centers throughout the province of British Columbia (BC), a Canadian province with a population of 4.4 million. The BC Cancer Agency (BCCA) is responsible for funding all systemic cancer therapy and is the sole provider of radiotherapy in $\mathrm{BC}$. All patients in the province who require radiation therapy for a diagnosis of SCC anal cancer are referred to the BCCA for consultation and treatment delivery.

Chemoradiation was delivered according to GICART, a standardized protocol, introduced in February 2010 and posted on the BCCA website [20]. Eligibility criteria for this therapy include a diagnosis of stage I-III squamous cell or cloacogenic carcinoma of the anal canal and ECOG performance status of less than or equal to 2 . Patients also need to have an adequate marrow reserve (ANC greater than or equal to $1.5 \times 10^{9} / \mathrm{L}$, platelets greater than $100 \times 10^{9} / \mathrm{L}$ ), with adequate renal (creatinine less than or equal to $1.5 \times \mathrm{ULN}$ ) and liver function (bilirubin less than or equal to $26 \mu \mathrm{mol} / \mathrm{L}$; AST/Alkaline Phosphatase less than or equal to $5 \times \mathrm{ULN}$ ).

Patients treated under the GICART protocol received a combination of chemotherapy and radiotherapy. Continuous radiation at a dose of 50.4 Gy in 28 fractions over $51 / 2$ weeks was recommended. Chemotherapy with Cap was delivered twice a day at a dose of $825 \mathrm{mg} / \mathrm{m}^{2}$ on days that radiotherapy was administered (days 1-5, 8-12, 15$19,22-26,29-33,36-40)$, to a total daily dose of $1650 \mathrm{mg} / \mathrm{m}^{2}$. As specified in the GICART protocol, Cap was administered orally with food, with the second dose administered 10-12 hours after the first. MMC is administered on day one week one intravenously, at a dose of $12 \mathrm{mg} / \mathrm{m}^{2}$ to a maximum dose of $20 \mathrm{mg}$.

\section{Study cohort selection and data extraction}

All SCC anal cancer patients diagnosed at BCCA and treated with GICART protocol from the time of its introduction in February 1, 2010 until March 29, 2012 were included in this study.

The charts from all SCC anal canal patients in this cohort were used to determine variations in chemotherapy doses, differences in radiation dosing and type of radiation technique delivered to each patient. Patient outcomes including relapses and deaths were documented until April 2013. To determine the levels of toxicity experienced by each patient in the cohort, a review was conducted of all the narratives dictated by the oncologist assigned to each patient. Nausea, vomiting, stomatitis and diarrhea toxicities were graded according to the GICART protocol. Dermatitis data was extrapolated from the narratives provided by the physicians and a graded according to the Dermatitis Grading Scale from the BCCA Care of Radiation Skin Reactions standard [21]. The most severe toxicity described for each patient was used in each case. For example, if a patient had grade 3 diarrhea but was experiencing grade 4 levels of nausea, they were classified as experiencing a grade 4 toxicity.

\section{Sources of research support}

The Provincial Pharmacy Database was used to identify eligible patients, and patient and tumor characteristics were identified through the BCCA Gastrointestinal Cancer Outcomes Unit (GICOU). The GICOU database prospectively documents standard pathologic and clinical criteria of patients referred to the BCCA. The study was conducted as a quality assurance initiative and was reviewed by the BCCA-University of British Columbia Research Ethics Board.

\section{Results}

\section{Patient characteristics}

The characteristics and diagnostic status of the $66 \mathrm{pa}-$ tients included in this study are shown in Table 1 . The majority of patients were female (62\%), HIV negative (99\%), non-smokers (74\%) and non-drinkers (74\%). Most patients $(n=61,93 \%)$ presented with an ECOG status between $0-1$, while three (5\%) presented with an ECOG 


\begin{tabular}{|c|c|}
\hline Characteristics & Number of patients (percent) \\
\hline Median age & 60 years $(44-82)$ \\
\hline \multicolumn{2}{|l|}{ Sex } \\
\hline Male & $25(38 \%)$ \\
\hline Female & $41(62 \%)$ \\
\hline \multicolumn{2}{|l|}{ HIV status } \\
\hline Positive & $1(2 \%)$ \\
\hline Negative & $65(99 \%)$ \\
\hline \multicolumn{2}{|l|}{ Smoking status } \\
\hline Smoker (have ever) & $17(26 \%)$ \\
\hline Non-smoker & $49(74 \%)$ \\
\hline \multicolumn{2}{|l|}{ Alcohol consumption } \\
\hline Drinker & $17(26 \%)$ \\
\hline Non-drinker & $49(74 \%)$ \\
\hline \multicolumn{2}{|l|}{ ECOG status } \\
\hline 0 & $42(64 \%)$ \\
\hline 1 & $19(29 \%)$ \\
\hline 2 & $3(5 \%)$ \\
\hline 3 & $2(3 \%)$ \\
\hline 4 & $0(0 \%)$ \\
\hline \multicolumn{2}{|l|}{ Stage of tumor } \\
\hline I & $26(39 \%)$ \\
\hline II & $15(23 \%)$ \\
\hline III & $25(38 \%)$ \\
\hline IV & $0(0 \%)$ \\
\hline \multicolumn{2}{|l|}{ Histology } \\
\hline Squamous cell carcinoma & $49(74 \%)$ \\
\hline Squamous carcinoma (keratinizing) & $8(12 \%)$ \\
\hline Squamous cell carcinoma (basaloid) & $5(8 \%)$ \\
\hline Tubulovillous adenoma & $2(3 \%)$ \\
\hline Adenocarcinoma & $2(3 \%)$ \\
\hline
\end{tabular}

Characteristics of all 66 patients included in the study are shown above, both as a total number and as a percentage of study participants.

status of 2 and two patients (3\%) had an ECOG status of 3. Staging was varied, as 26 patients (39\%) presented with a stage I tumor, 15 (23\%) presented with a stage II tumor and 25 (38\%) presented with a stage III tumor.

Histologically, the tumours were classified as follows: of 66 patients, 62 (94\%) had squamous cell carcinoma of which eight were described as keratinizing, and five as basaloid. Two patients (3\%) had tubulovillous adenoma, and an additional two patients (3\%) had adenocarcinoma. All 66 patients were included in the dose intensity and toxicity analysis, while only patients with SCC (62) were included in the outcome analysis.

\section{Treatment dose intensity}

All patients included in the study initiated therapy with both capecitabine and mitomycin $\mathrm{C}$. The doses administered are shown in Table 2, along with the number of dose reductions, increases, omissions, and discontinuations for each therapy administered.

Patients received a median dose of $825 \mathrm{mg} / \mathrm{m}^{2}$ of Cap administered twice daily on radiation days. Dose reductions were recorded for 13 patients (20\%) due to apparent toxicity during treatment. There were no recorded increases, omissions or discontinuations for this drug. The median duration of therapy of capecitabine was five and a half weeks on week-days, with a treatment range from three to six weeks. Seven patients initiated therapy at a lower dose of Cap $\left(500-749 \mathrm{mg} / \mathrm{m}^{2}\right)$ due to varying issues ranging from patient comorbidities, previous chemotherapy treatment, and the use of therapy purely for palliative purposes. None of the patients starting at this lower dose required a subsequent dose reduction.

The median dose of MCC delivered was $12 \mathrm{mg} / \mathrm{m}^{2}$ on day one, week one. Pre-planned dose reductions were recorded for three (5\%) of patients, of which two cases were due to patient co-morbidities and one was due to an infusion reaction.

The radiation dose, technique and treatment duration received by patients in this cohort is shown in Table 3. Median planned dose of radiation was 51.9 Gy over a median of 5.5 weeks, range 25.0 to $63 \mathrm{~Gy}$. Comparing the planned versus delivered radiotherapy doses, we can see that the majority (98\%) of patients received the planned dose of radiation. There was one recorded dose reduction, from 60 Gy to 12 Gy due to radiation complications (early moist desquamation).

Table 2 Chemotherapy dosings $(n=66)$ as a total number and as a percentage

\begin{tabular}{|c|c|}
\hline Capecitabine dose administered & Number of patients (percent) \\
\hline \multicolumn{2}{|c|}{ Number of patients starting at full dose $\left(750-825 \mathrm{mg} / \mathrm{m}^{2}\right)$} \\
\hline - No subsequent dose reduction & $59(78 \%)$ \\
\hline - Dose reduction & $13(22 \%)$ \\
\hline - Discontinuation & $0(0 \%)$ \\
\hline \multicolumn{2}{|c|}{ Number of patients starting at dose level $-1\left(500-749 \mathrm{mg} / \mathrm{m}^{2}\right)$} \\
\hline - Received & $7(11 \%)$ \\
\hline - Dose reduction & $0(0 \%)$ \\
\hline - Discontinuation & $0(0 \%)$ \\
\hline Mean weeks of capectiabine & $5.5(2.5-6)$ \\
\hline Mitomycin dose administered & Number of patients (percent) \\
\hline - Full dose $\left(12 \mathrm{mg} / \mathrm{m}^{2}\right)$ & $63(95 \%)$ \\
\hline - Dose level -1 & $3(5 \%)$ \\
\hline
\end{tabular}

The capecitabine and mitomycin dose administered to patients in the study, both as a total number and as a percentage of study participants. 
Table 3 Radiation delivery method and dose received by patients in study $(n=66)$

\begin{tabular}{llc}
\hline Radiation technique & $\begin{array}{c}\text { Number of patients } \\
\text { treated } \mathbf{n}(\%)\end{array}$ \\
\hline 3D-CRT & $50(76 \%)$ \\
IMRT & $16(24 \%)$ \\
Radiation dose planned $\mathbf{n ( \% )}$ & $\begin{array}{c}\text { Number of patients } \\
\text { with dose reduction }\end{array}$ \\
10-15 Gy & $0(0 \%)$ & 0 \\
15-20 Gy & $0(0 \%)$ & 0 \\
20-25 Gy & $0(0 \%)$ & 0 \\
$25-30$ Gy & $3(5 \%)$ & 0 \\
$30-35$ Gy & $1(2 \%)$ & 0 \\
$35-40$ Gy & $0(0 \%)$ & 0 \\
$40-45$ Gy & $1(2 \%)$ & 0 \\
$45-50$ Gy & $6(9 \%)$ & 0 \\
$50-55$ Gy & $45(70 \%)$ & 1 (Reduced to 12 Gy) \\
$55-60$ Gy & $9(14 \%)$ & 0 \\
$60-65$ Gy & $1(2 \%)$ &
\end{tabular}

Median: $51.9 \mathrm{~Gy}$

Range: 25.0-63 Gy

Total: 1 (out of 66) (2\%)

The radiation dose received by patients in the study and dose reduction, both as a total number and as a percentage of study participants. Abbreviations: 3D-CRT 3-Dimensional Conformal Radiation Therapy; IMRT Intensity Modulated Radiation Therapy; Gy gray.

Radiation techniques were also examined. Threedimensional conformal RT (3D-CRT) was shown to be the primary technique used, with 50 patients $(76 \%)$ receiving this treatment, while the remaining 16 patients $(24 \%)$ received intensity-modulated RT (IMRT) (Table 3).

\section{Patient toxicities}

Toxicity grades adverse events experienced by patients are described in Table 4 and include nausea, vomiting and stomatitis. A total of 54 patients (82\%) experienced grades $0-1$ toxicity, including minor changes in bowel habits, one episode of nausea and vomiting per day as well as the presence of painless ulcers, erythema or mild soreness. A total of seven patients (11\%) experienced grade 2 toxicity, including moderate changes in bowel habits, 2-5 episodes of nausea and vomiting a day and painful erythema, edema or ulcers. Two patients $(3 \%)$ experience grade diarrhea and three patients (5\%) were classified as having grade 4 diarrhea.

Peri-anal radiation dermatitis was experienced in varying degrees by numerous patients during the course of treatment (Table 5). Using the Dermatitis Grading Scale from the BCCA Care of Radiation Skin Reactions [21], there were a total of 19 patients (29\%) that experienced grade 1 toxicities which encompassed minor skin changes such as numbness and tingling. Five patients (8\%) experienced a grade two toxicity noted by erythema and swelling,
Table 4 Adverse events experienced by patients in the study $(n=66)$

\begin{tabular}{|c|c|}
\hline $\begin{array}{l}\text { Toxicity grade of } \\
\text { adverse event }\end{array}$ & $\begin{array}{l}\text { Number of patients } \\
\text { (percent) } \mathrm{N}=66\end{array}$ \\
\hline 0 & $35(53 \%)$ \\
\hline 1 & 19 (29\%) \\
\hline 2 & $7(11 \%)$ \\
\hline 3 & $2(3 \%)$ \\
\hline 4 & $3(5 \%)$ \\
\hline \multicolumn{2}{|c|}{$\begin{array}{l}\text { Case descriptions of grade } 3 \text { and } 4 \text { toxicity description } \\
\text { (case by case) }\end{array}$} \\
\hline Case 1 , grade 3 & Grade 2 stomatitis, grade 3 diarrhea \\
\hline Case 2 , grade 3 & Grade 3 diarrhea, stool incontinence \\
\hline Case 3 , grade 4 & Grade 4 diarrhea \\
\hline Case 4 , grade 4 & Grade 4 diarrhea, stool incontinence \\
\hline Case 5 , grade 4 & Grade 4 diarrhea \\
\hline
\end{tabular}

and 42 patients (63\%) experienced a grade 3 toxicity highlighted by instances of moist desquamation and ulceration.

\section{Patient outcomes}

Median follow-up from time of initial diagnosis was 20 months. Of patients with Squamous cell histology $(\mathrm{N}=62) 94 \%$ had no evidence of relapse as of April 2013. Four patients (6\%), one stage I, one stage II and two stage III, had recorded relapses. One relapsed patient with initial stage II disease died, however, death was not attributed to treatment. Sorting the outcomes by cancer stage, one out of 26 patients (4\%) with stage I tumours experienced a local relapse. For patients with stage II tumors, one out of 15 patients experienced a distant relapse, with two recorded deaths not attributed to treatment, and one out of the 25 patients with stage III tumours experiencing a distant relapse. Among 4 patients with tubulovillous adenoma and adenocarcinoma histology, $0 \%$ experienced a relapse.

Table 5 Radiation dermatitis experienced by patients in this study, by toxicity grade $(n=66)$

\begin{tabular}{ll}
\hline Toxicity grade & Number of patients (percent) \\
\hline $\mathbf{1}$ & $19(29 \%)$ \\
$\mathbf{2}$ & $5(8 \%)$ \\
$\mathbf{3}$ & $42(63 \%)$ \\
\hline
\end{tabular}

The breakdown of radiation dermatitis experienced by patients during this study based on toxicity grade. 


\section{Discussion}

In this study, the charts of 66 patients with stage I-III SCC of the anal canal treated at BCCA with multimodality therapy - Cap, MMC and radiation - were retrospectively reviewed. Although the global standard of care for SCC of the anal canal is concurrent radiotherapy with 5-FU and $\mathrm{MMC}$, a standard protocol substituting infusional 5-FU with oral capecitabine was introduced in 2010 as an alternative non-infusional regimen [20]. Results of the study showed that while the majority of patients experienced some form of chemotherapy or radiation induced toxicity, protocol therapy was well tolerated; despite some dose reductions due to apparent toxicities there were no treatment discontinuations. The majority of patients who underwent this therapy since its initiation in 2010 currently have their tumors in remission with only a few reported cases of relapses and no treatment-related deaths.

Limitations encountered in this study centered on the retrospective nature of the collection of toxicity data. While the number of patients included was modest, the current study adds to the description of efficacy and outcomes of this regimen. Results compare favorably to the previous publication of the phase II study on which the current GICART protocol was based [19]. To our knowledge, there are no other prospective or retrospective studies describing this treatment regimen.

\section{Patient characteristics}

Median age of patients in this cohort was 60 and the majority of tumors were stage I-II at presentation. It was noteworthy that there were almost twice as many female subjects in the study cohort as males. A significant increase in SCC has been documented in both men and women [22]. Most study subjects did not smoke, and there was only one HIV-positive subject included in the cohort. The preferred institutional chemotherapy regimen for HIV-positive patients is cisplatin-capecitabine due to the more favorable hematological toxicity described with cisplatin over MMC [6].

\section{Chemotherapy tolerance}

Toxicity grades for nausea, vomiting and diarrhea were low despite the high dose intensity of chemotherapy drugs administered (Table 4). Most patients experienced only grade $0-1$ toxicities indicating favorable outcomes with low side effects, while 12 patients (19\%) experienced grade 2-4 toxicities for nausea, vomiting and diarrhea.

Overall, the starting doses of Cap (generally of $825 \mathrm{mg} / \mathrm{m}^{2}$ BID on each radiotherapy day) were well tolerated by the patients; however, $20 \%$ of patients required dose reductions, likely for reasons of apparent toxicity. A total of $8 \%$ of patients experienced grade 3-4 gastrointestinal toxicities which would lead to protocol-specified dose reductions.
11\% experienced grade 2 gastrointestinal and skin toxicities which would require a dose reduction on second occurrence.

The chart review revealed that while all of the patients involved in this study experienced some degree of dermatitis during radiotherapy treatment, only one patient required radiation dose reduction. Generally, radiation treatment of any degree causes some sort of reaction in the treated and neighboring skin layers so the results themselves are not unexpected given the duration and intensity of the radiation doses.

\section{Radiation therapy}

The GICART protocol specified 50.4 Gy in 28 fractions over 5.5 weeks. A range of treatment schedules, techniques and doses were used to reflect variability in individual patient factors and tumour characteristics. Generally, patients with a higher tumour stage or patients physically able to withstand higher doses of radiation were given larger radiation doses, while patients with lower stage tumors were treated with lower radiation doses. In a previous phase II study, escalating doses of radiation were prospectively defined in the following manner: patients with stage T1 tumours were given a dose of 45 Gy in 25 fractions, patients with stage T2 tumours were given a dose of 55 Gy in 30 fractions, and patients with stage T3-4 tumours were given 59 Gy in 32 fractions [23]. Radiation oncologists involved in the current study used similar criteria to justify dose variation according to tumor stage. There was no discernible difference in outcomes between the patients treated with the two radiation techniques, 3D-CRT and IMRT.

All patients included in the study achieved initial remission of disease. Subsequent relapses were infrequent and occurred in only $4 \%$ of stage I, $7 \%$ of stage II patients and in $6 \%$ of patients with stage III disease. Due to limited follow-up time, no conclusion regarding the efficacy of the regimen can be made at this time. However, phase III evidence from other disease settings show equivalence of capecitabine to infusional 5 -FU in multiple settings [18,24,25]. One randomized head-to-head study of infusional 5-FU versus capecitabine in combination with radiation for stage II/III rectal cancer demonstrated equivalent efficacy and toxicity when given with pelvic radiation at doses of 50.4 Gy over 28-31 days [16,17].

No significant differences in the rates of pathologic complete response or grade 3-4 diarrhea were identified between the infusional 5-FU and versus CAP at a dose of $825 \mathrm{mg} / \mathrm{m}^{2}$ twice daily, 5 days/wk. Given the favorable tolerance and high dose intensity for both capecitabine and radiation described in the current study, it is unlikely that the substitution capecitabine for infusional 5-FU in 
the adjuvant treatment of SCC will result in inferior outcomes.

In this study there was a wide variation in radiation doses. At this point in time, the variations in administered radiation doses do not correlate with different outcomes, suggesting that lower radiation doses may be considered in some patients. Longer term follow up of patients in this cohort is necessary to ensure that patients who received lower doses of radiation and chemotherapy have similar outcomes to those who received larger doses. Our intention is to follow this cohort to see if additional correlations between radiation and chemotherapy and long term survival outcomes can be made.

\section{Conclusions}

Combined modality therapy of capecitabine plus mitomycin and radiotherapy is well tolerated and allows high dose intensity of radiation and chemotherapy in a population based setting. Substitution of capecitabine for infusional 5-FU is feasible and may be a reasonable consideration for patients and physicians who prefer to avoid the inconvenience and potential complications of a central infusional device. A range of planned radiation doses, schedules and techniques were employed reflecting tumor and patient characteristics. Prospective studies to determine optimal radiation dose would be justified.

\section{Abbreviations}

APR: Abdominoperineal resection; BCCA: British Columbia Cancer Agency; Cap: Capecitabine; HPV: Human papilloma virus; IMRT: Intensity-modulated RT; MMC: Mitomycin C; RT: Radiation therapy; SCC: Squamous cell carcinoma; 3D-CRT: Three-dimensional conformal RT; 5-FU: 5-fluorouracil.

\section{Competing interests}

None of the authors have any conflicts of interest to report. Research support for this study was provided by the British Columbia Cancer Agency.

\section{Authors' contributions}

All authors, GT, BJ, MF and HFK, conceptualized the project, extracted and analysed data, and prepared the tables and manuscript. All authors read and approved the final manuscript.

\section{Acknowledgments}

The authors wish to thank Chrystal Palaty for assistance in preparing the manuscript for publication.

\section{Author details}

${ }^{1}$ Royal College of Surgeons in Ireland, BC Cancer Agency, Fraser Valley Cancer Center, Surrey, BC, Canada. ${ }^{2}$ Division of Medical Oncology, BC Cancer Agency, Fraser Valley Cancer Center, Surrey, BC, Canada. ${ }^{3}$ Division of Radiation Oncology, Simcoe Muskoka Regional Cancer Centre, Barrie, ON, Canada. ${ }^{4}$ Division of Medical Oncology, British Columbia Cancer Agency Vancouver Center, Vancouver, BC V5Z 4E6, Canada.

Received: 29 December 2013 Accepted: 9 April 2014

Published: 29 May 2014

\section{References}

1. Siegel R, Ma J, Zou Z, Jemal A: Cancer statistics, 2014. CA Cancer J Clin 2014, 64(1):9-29

2. Bilimoria $K Y$, Bentrem DJ, Rock CE, Stewart $A K, K o C Y$, Halverson A: Outcomes and prognostic factors for squamous-cell carcinoma of the anal canal: analysis of patients from the national cancer data base. Dis Colon Rectum 2009, 52(4):624-631.

3. Ryan DP, Mayer RJ: Anal carcinoma: histology, staging, epidemiology, treatment. Curr Opin Oncol 2000, 12(4):345-352.

4. Moscicki AB, Schiffman M, Burchell A, Albero G, Giuliano AR, Goodman MT, Kjaer SK, Palefsky J: Updating the natural history of human papillomavirus and anogenital cancers. Vaccine 2012, 20(30):089.

5. Poggio J: Premalignant lesions of the anal canal and squamous cell carcinoma of the anal canal. Clin Colon Rectal Surg 2011, 24(03):177-192.

6. James RD, Glynne-Jones R, Meadows HM, Cunningham D, Myint AS, Saunders MP, Maughan T, McDonald A, Essapen S, Leslie M, Falk S, Wilson C, Gollins S, Begum R, Ledermann J, Kadalayil L, Sebag-Montefiore D: Mitomycin or cisplatin chemoradiation with or without maintenance chemotherapy for treatment of squamous-cell carcinoma of the anus (ACT II): a randomised, phase 3, open-label, $2 \times 2$ factorial trial. Lancet Oncol 2013, 14(6):516-524.

7. Epidermoid anal cancer: results from the UKCCCR randomised trial of radiotherapy alone versus radiotherapy, 5-fluorouracil, and mitomycin. UKCCCR anal cancer trial working party. UK co-ordinating committee on cancer research. Lancet 1996, 348(9034):1049-1054.

8. Bartelink H, Roelofsen F, Eschwege F, Rougier P, Bosset JF, Gonzalez DG, Peiffert D, van Glabbeke M, Pierart M: Concomitant radiotherapy and chemotherapy is superior to radiotherapy alone in the treatment of locally advanced anal cancer: results of a phase III randomized trial of the European organization for research and treatment of cancer radiotherapy and gastrointestinal cooperative groups. J Clin Oncol 1997, 15(5):2040-2049.

9. Northover J, Glynne-Jones R, Sebag-Montefiore D, James R, Meadows H, Wan S, Jitlal M, Ledermann J: Chemoradiation for the treatment of epidermoid anal cancer: 13-year follow-up of the first randomised UKCCCR anal cancer trial (ACT I). Br J Cancer 2010, 102(7):1123-1128.

10. Flam M, John M, Pajak TF, Petrelli N, Myerson R, Doggett S, Quivey J, Rotman M, Kerman H, Coia L, Murray K: Role of mitomycin in combination with fluorouracil and radiotherapy, and of salvage chemoradiation in the definitive nonsurgical treatment of epidermoid carcinoma of the anal canal: results of a phase III randomized intergroup study. J Clin Oncol 1996, 14(9):2527-2539.

11. Cummings BJ, Keane TJ, O'Sullivan B, Wong CS, Catton CN: Epidermoid anal cancer: treatment by radiation alone or by radiation and 5-fluorouracil with and without mitomycin C. Int J Radiat Oncol Biol Phys 1991, 21(5):1115-1125.

12. Peiffert D, Seitz JF, Rougier P, Francois E, Cvitkovic F, Mirabel X, Nasca S, Ducreux M, Hannoun-Levi JM, Lusinchi A, Debrigode E, Conroy T, Pignon JP, Gerard JP: Preliminary results of a phase II study of high-dose radiation therapy and neoadjuvant plus concomitant 5 -fluorouracil with CDDP chemotherapy for patients with anal canal cancer: a French cooperative study. Ann Oncol 1997, 8(6):575-581.

13. Martenson JA, Lipsitz SR, Wagner H Jr, Kaplan EH, Otteman LA, Schuchter LM, Mansour EG, Talamonti MS, Benson AB 3rd: Initial results of a phase II trial of high dose radiation therapy, 5 -fluorouracil, and cisplatin for patients with anal cancer (E4292): an eastern cooperative oncology group study. Int J Radiat Oncol Biol Phys 1996, 35(4):745-749.

14. Ajani JA, Winter KA, Gunderson LL, Pedersen J, Benson AB 3rd, Thomas CR Jr, Mayer RJ, Haddock MG, Rich TA, Willett C: Fluorouracil, mitomycin, and radiotherapy vs fluorouracil, cisplatin, and radiotherapy for carcinoma of the anal canal: a randomized controlled trial. JAMA 2008, 299(16):1914-1921.

15. Gunderson LL, Winter KA, Ajani JA, Pedersen JE, Moughan J, Benson AB 3rd, Thomas CR Jr, Mayer RJ, Haddock MG, Rich TA, Willett CG: Long-term update of US GI intergroup RTOG 98-11 phase III trial for anal carcinoma: survival, relapse, and colostomy failure with concurrent chemoradiation involving fluorouracil/mitomycin versus fluorouracil/cisplatin. J Clin Oncol 2012, 30(35):4344-4351.

16. Allegra CJ, Yothers G, O'Connell MJ, Roh MS, Beart RW, Petrelli NJ, Lopa SH, Sharif S, Wolmark N: Neoadjuvant Therapy for Rectal Cancer: Mature Results from NSABP Protocol R-04, Abstract 390. I Clin Oncol 2014, 32(suppl 3; abstr 390).

17. Roh MS, Yothers GA, O'Connell MJ, Beart RW, Pitot HC, Shields AF, Parda DS, Sharif S, Allegra CJ, Petrelli NJ, Landry JC, Ryan DP, Arora A, Evans TL, Soori GS, Chu L, Landes RV, Mohiuddin M, Lopa S, Wolmark N: The impact of capecitabine and oxaliplatin in the preoperative multimodality 
treatment in patients with carcinoma of the rectum: NSABP R-04. J Clin Oncol 2011, 29(suppl; abstr 3503):2011.

18. De Paoli A, Chiara S, Luppi G, Friso ML, Beretta GD, Del Prete S, Pasetto L, Santantonio M, Sarti E, Mantello G, Innocente R, Frustaci S, Corvò R, Rosso R: Capecitabine in combination with preoperative radiation therapy in locally advanced, resectable, rectal cancer: a multicentric phase II study. Ann Oncol 2006, 17(2):246-251.

19. Glynne-Jones R, Meadows H, Wan S, Gollins S, Leslie M, Levine E, McDonald AC, Myint S, Samuel L, Sebag-Montefiore D: EXTRA-a multicenter phase II study of chemoradiation using a 5 day per week oral regimen of capecitabine and intravenous mitomycin C in anal cancer. Int J Radiat Oncol Biol Phys 2008, 72(1):119-126.

20. British Columbia Cancer Agency Protocol Summary for Curative Combined Modality Therapy for Carcinoma of the Anal Canal Using Mitomycin, Capecitabine and Radiation Therapy (GICART). In Edited by. 2013. http://www.bccancer.bc.ca/NR/rdonlyres/COA8933B-3AAD-4EBAA95C-4552A0A060C9/63672/GICART_Protocol_1May2013.pdf. (Accessed May 30, 2013).

21. British Columbia Cancer Agency Care of Radiation Skin Reactions. In Edited by. 2012. http://www.bccancer.bc.ca/NR/rdonlyres/79E81484-680941CF-8CC2-0646DA6003F8/64011/CareofRadiationSkinReactions.pdf (Accessed July 22, 2013).

22. Grulich AE, Poynten IM, Machalek DA, Jin F, Templeton DJ, Hillman RJ: The epidemiology of anal cancer. Sex Health 2012, 9(6):504-508.

23. Eng C, Chang GJ, Das P, Rodriguez-Bigas MD, Skibber JM, Qiao W, Rosner GL, Ukegbu LT, Wolff AR, Crane CH: Phase II study of capecitabine and oxaliplatin with concurrent radiation therapy (xelox-xrt) for squamous cell carcinoma of the anal canal. J Clin Oncol 2009, 27(No 15 S (May 20 Supplement)):4116.

24. Haller DG, Tabernero J, Maroun J, de Braud F, Price T, Van Cutsem E, Hill M, Gilberg F, Rittweger K, Schmoll HJ: Capecitabine plus oxaliplatin compared with fluorouracil and folinic acid as adjuvant therapy for stage III colon cancer. J Clin Oncol 2011, 29(11):1465-1471.

25. Twelves C, Wong A, Nowacki MP, Abt M, Burris H 3rd, Carrato A, Cassidy J, Cervantes A, Fagerberg J, Georgoulias V, Husseini F, Jodrell D, Koralewski P, Kroning H, Maroun J, Marschner N, McKendrick J, Pawlicki M, Rosso R, Schuller J, Seitz JF, Stabuc B, Tujakowski J, Van Hazel G, Zaluski J, Scheithauer W: Capecitabine as adjuvant treatment for stage III colon cancer. N Engl J Med 2005, 352(26):2696-2704.

doi:10.1186/1748-717X-9-124

Cite this article as: Thind et al: Chemoradiation with capecitabine and mitomycin-C for stage I-III anal squamous cell carcinoma. Radiation Oncology 2014 9:124.

\section{Submit your next manuscript to BioMed Central and take full advantage of:}

- Convenient online submission

- Thorough peer review

- No space constraints or color figure charges

- Immediate publication on acceptance

- Inclusion in PubMed, CAS, Scopus and Google Scholar

- Research which is freely available for redistribution 